\title{
Competencias transversales y profesionalismo en la carrera de Medicina en Argentina. De las prescripciones al aula
}

\section{Transversal competences and professionalism in Argentina's medicine school. From prescriptions to the classroom.}

\author{
Marta A. Tenutto Soldevilla ${ }^{1}$ \\ 1.Docente del Doctorado en Educación de la Universidad Católica de Córdoba. Docente del Profesorado de la Universidad Isalud. Asesora Pedagógica de la \\ Fundación Hospital de Pediatría Prof. Juan P. Garrahan. \\ Correspondencia: Marta Alicia Tenutto Soldevilla; email: marta.tenutto@gmail.com.
}

\section{Resumen}

Los médicos y médicas intervienen en el despliegue vital de niños, niñas, jóvenes y adultos con decisiones que pueden marcar no solo la calidad de vida de las personas sino, particularmente, la frontera entre la vida y la muerte.

En las últimas décadas, se han desarrollado diversas investigaciones sobre las consecuencias de la mala praxis, la iatroegenia y los errores médicos. Se trata de prácticas señaladas como la tercera causa de muerte en los Estados Unidos de Norteamérica ${ }^{1}$. Estos casos muestran que los conocimientos y competencias específicas resultan insuficientes para un buen desempeño profesional y remiten a problemas de otro orden como dificultades en la comunicación, así como problemas para trabajar en equipo, entre otros. Es decir, aluden a competencias profesionales transversales.

En ese marco se desarrolló la tesis doctoral de la autora que se presenta en una versión sintética en este artículo. En dicha investigación se indagó en qué medida se prescribe su enseñanza, se enseñan y se evalúan las competencias profesionales transversales y el profesionalismo en la carrera de Medicina en Argentina. Para concretar el estudio, se analizaron documentos de políticas educativas en la versión de las resoluciones y documentos del caso seleccionado para este estudio, también se aplicaron encuestas a estudiantes $(\mathrm{N}=140)$ y profesionales que se desempeñan en centros de salud $(\mathrm{N}=100)$. En este artículo se presentarán los resultados de las escalas de las Políticas educativas y del aula con mayor profundidad que los resultados de la institución por cuestiones relativas a la extensión del texto. Para el análisis de los datos se utilizó el método interpretativo.

Palabras clave: competencias profesionales transversales, profesionalismo, medicina.

\begin{abstract}
The physicians intervene in the vital deployment of children, young people and adults with decisions that can mark not only the quality of life of people but, in particular, the border between life and death. In recent decades, various investigations have been developed on the consequences of malpractice, iatrogenesis and medical errors. These are practices indicated as the third cause of death in the United States of America ${ }^{1}$. Hese cases show that specific knowledge and skills are insufficient for good professional performance and refer to problems of another order such as communication difficulties as well as problems to work in teams, among others. That is, they refer to transversal professional competences.In that framework, the author's doctoral thesis was presented, which is presented in a synthetic version in this article. In this research it was investigated to what extent their teaching is prescribed in the curriculum, cross-cutting professional
\end{abstract}

Revista Methodo: Investigación Aplicada a las Ciencias Biológicas. Universidad Católica de Córdoba. Jacinto Ríos 571 Bo Gral. Paz. X5004FXS. Córdoba. Argentina. Tel.: (54) 3514517299 / Correo: methodo@ucc.edu.ar / Web: methodo.ucc.edu.ar | ARTICULO ORIGINAL Rev. Methodo 2020;5(1):09-14 
competencies and professionalism in the medical career in Argentina are taught and evaluated. To finalize the study, educational policy documents were analyzed in the resolution version, documents of the case selected for this study were analyzed and surveys were applied to students $(\mathrm{N}=140)$ and professionals working in health centers $(\mathrm{N}=100)$. This article will present the results of the scales of the Educational Policies and the classroom more deeply than the results of the institution for issues related to the length of the text. For the analysis of the data the interpretive method was used.

Keywords: teaching and evaluation, transversal professional competences, medicine.

\section{Introducción}

Renovados desafíos se presentan a médicos y médicas en la actualidad. Desde el chamanismo primera práctica vinculada con la Medicinapasando por el nacimiento de la Medicina científica del siglo XVIII, la sociedad ha experimentado modificaciones sustanciales que atraviesan su formación y sus prácticas.

Los profesionales de la salud intervienen en el despliegue vital de los seres humanos con decisiones que pueden marcar no solo su calidad de vida sino, particularmente, la frontera entre la vida y la muerte. En las últimas décadas, se han desarrollado diversas investigaciones sobre las consecuencias de la mala praxis, los errores médicos y la iatrogenia. (Tabla 1).

Tabla 1. Definición de términos

\begin{tabular}{|c|c|}
\hline Palabras & Significado \\
\hline Mala praxis & $\begin{array}{l}\text { Se llama a aquello que provoca un daño parcial o total en } \\
\text { el cuerpo o en la salud de la persona humana como } \\
\text { consecuencia de un accionar profesional realizado con } \\
\text { imprudencia, negligencia o impericia. Se denomina: 1) } \\
\text { impericia a la falta de conocimientos en determinado arte } \\
\text { o profesión; 2) imprudencia el no tomar precauciones para } \\
\text { evitar un riesgo o actuar en forma precipitada y 3) } \\
\text { negligencia es lo opuesto al sentido del deber, los médicos } \\
\text { tienen la obligación de llevar constancia escrita de sus } \\
\text { actos profesionales. Fuente: Fondo de Resguardo } \\
\text { Profesional. Colegio de Médicos de la Provincia de } \\
\text { Buenos Aires, Región III. }\end{array}$ \\
\hline Errores médicos & $\begin{array}{l}\text { Se llama a los efectos adversos o consecuencias } \\
\text { indeseadas del proceso de atención médica, sin "mala fe" } \\
\text { (2) }\end{array}$ \\
\hline Iatrogenia & $\begin{array}{l}\text { Deriva de iatrogénesis (iatros/ médico en griego, y } \\
\text { génesis: crear) que significa "provocado por el médico o } \\
\text { sanador }\end{array}$ \\
\hline
\end{tabular}

Estas investigaciones muestran que los conocimientos y competencias específicas resultan insuficientes para un buen desempeño profesional. En los Estados Unidos de América cada 75000 operaciones se produce una intervención quirúrgica en el lugar erróneo o en un paciente equivocado $^{3}$. Y, según las investigaciones relevadas por otro autor, los errores médicos constituyen la tercera causa de muerte en ese país ${ }^{1}$. Abordar la formación médica es una tarea bifronte. Por un lado, dirige su mirada hacia la formación de grado y posgrado, y por el otro al sistema de salud, espacios que se despliegan en una sociedad que se desarrolla en un tiempo y territorio determinados. En este sentido, las características de los servicios de salud presentadas en la década de 1980, se han hecho realidad. Estas características son: 1) aumento de la demanda de servicios sociosanitarios de compleja financiación causado por el envejecimiento de la población, 2) disminución del hospitalocentrismo, aumento de los bolsones de pobreza, la exclusión y la conflictividad social y 3) e instalación del mercado sanitario abierto y competitivo que puede convertirse en un "mercado de la salud" .

Ahora bien, si en cambio, la mirada se coloca sobre la formación de médicos y médicas, surgen diversos cuestionamientos. Se afirma que la propuesta resulta excesivamente teórica, ofrece una metodología poco activa, posee evaluaciones centradas en los contenidos, con planes de estudio que excluyen la bioética y el profesionalismo ${ }^{5} \mathrm{y}$ brinda una insuficiente formación en competencias específicas y/o genéricas ${ }^{6}$. La educación profesional del médico debería favorecer la capacidad de resolver problemas y que "el conjunto de médicos que estamos formando es malo, los indicadores lo muestran"7.

Por otro lado, para comprender los procesos curriculares resulta necesario, analizar las diversas escalas de la gestión ${ }^{8}$. Por ello, en esta investigación se analizan las escalas de las Políticas educativas, de la institución y del aula. En ese contexto, la pregunta que guía esta indagación es: ¿cómo son definidas y cómo se desarrolla la formación de las competencias profesionales transversales y el profesionalismo desde la escala de las políticas educativas hasta la escala del aula en la carrera de Medicina en Argentina?

Se aborda la formación médica desde una perspectiva integral y, por ello, se ha optado por las competencias transversales en tanto transferibles, es decir, se ponen en juego en diferentes ámbitos profesionales ${ }^{9}$, y les permiten desempeñarse en contextos diversos con autonomía, flexibilidad, ética, responsabilidad ${ }^{10}$. Se incluye el término "profesionales" al de competencias transversales, para explicitar la

Revista Methodo: Investigación Aplicada a las Ciencias Biológicas. Universidad Católica de Córdoba. Jacinto Ríos 571 Bo Gral. Paz. X5004FXS. Córdoba. Argentina. Tel.: (54) 3514517299 / Correo: methodo@ucc.edu.ar / Web: methodo.ucc.edu.ar | ARTICULO ORIGINAL Rev. Methodo 2020;5(1):09- 
posición asumida. Las competencias transversales pueden ser abordadas desde, por lo menos, dos enfoques. El primero, en un sentido genérico, como "competencias para la vida", propias de los niveles educativos iniciales o básicos y el segundo - adoptado en esta investigación- como aquellas que integran los aprendizajes de las profesiones. Se incluye al profesionalismo como una exigencia de desempeño que se encuentra integrado por conocimientos y competencias específicas y, en particular, por el compromiso ético y social. Además, en ocasiones, la propuesta de formación del profesionalismo se solapa con la de competencias, por lo que no es posible eludir el tema.

Objetivos: Describir y explicar la formación de competencias profesionales transversales y el profesionalismo desde la escala de las políticas educativas hasta la escala del aula en la carrera de Medicina en Argentina.

\section{Población y métodos}

Se optó por la formación del/la médico/a porque es una profesión entronizada por la sociedad desde hace siglos, sociedad que les asigna el lugar del saber y porque son ello/as quienes, al asumir ese lugar de autoridad, se constituyen en productores y reproductores de significados (ver nota: a) que atraviesan la trama social. Se trata de una profesión que se hace escuchar -como dice Bourdieu ${ }^{11}$ - en tanto lenguaje autorizado.

Se ha elegido la carrera de Medicina, en virtud de dos criterios: accesibilidad y efectos de esta formación en el despliegue vital de los sujetos, como fue mencionado precedentemente. Los criterios mencionados se aplican al caso seleccionado la facultad de Medicina de una universidad pequeña de reciente creación.

En la escala de las políticas educativas se ha optado por analizar las resoluciones que regularon la formación del futuro profesional médico, en particular, aquellas que constituyeron los referentes para las convocatorias de acreditación de la carrera. El corpus estuvo compuesto por: Resolución del Ministerio de Cultura y Educación (MCyE) $\mathrm{N}^{\mathrm{o}} 535^{12}$ y Resolución del Ministerio de Educación, Ciencia y Tecnología (MECyT) No $1314^{13}$.

En relación con la escala de la institución, se trata de un estudio de caso. La decisión se fundamenta en que este tipo de estudio permite abordar un fenómeno contemporáneo dentro de un contexto de la vida real ${ }^{14}$. A la crítica clásica de que las conclusiones del estudio de caso no son generalizables, se responde que, por el contrario, sus resultados sí pueden generalizarse a otros que representen condiciones teóricas similares porque no se trata de una generalización estadística sino de una generalización analítica ${ }^{15}$.En esta escala, se analizaron los documentos de la institución. En este artículo solo se hará una mención a ellos.

En relación con la escala del aula, se aplicaron encuestas a estudiantes $(\mathrm{N}=140)$ y profesionales $(\mathrm{N}=100)$. Para la conformación de la muestra se trabajó con la técnica de bola de nieve. En la encuesta se indagaron aquellos atributos que tipifican al profesionalismo, así como las competencias profesionales transversales tomadas de Tuning Latinoamérica, los espacios curriculares en que se enseñan, qué y cómo se enseñan y se evalúan.

El enfoque desde el cual se analizaron los datos es el interpretativo. Sus iniciadores fueron teólogos protestantes que. en el siglo XVI, lo emplearon para la interpretación de la Biblia. Siglos más tarde, fue impulsado por la antropología y la psicología. El objetivo de este método es profundizar y generalizar el conocimiento acerca de por qué las personas, grupo y sociedad en general actúan del modo en que lo hacen.

\section{Resultados}

Las normativas son discursos reglados, prescriptivos, de carácter legal y político. En el caso de estas resoluciones, ostentan un triple atravesamiento: el determinado por las características de un tratamiento jurídico, el del marco educativo en general y el de la educación médica en particular. En la Res. MCyE. $\mathrm{N}^{\mathrm{0}} 535^{12}$, no se mencionan las competencias ni el profesionalismo, sí las capacidades. Se entronizan los conocimientos considerados científicos. En la resolución MECyT $\mathrm{N}^{\mathrm{o}} 1314^{13}$, se enuncian competencias del médico, es decir del egresado. Se trata de un modelo mixto donde figuran competencias y contenidos de las áreas disciplinares. Las competencias se encuentran centradas en la obtención de un logro individual. La dimensión dedicada a Profesionalismo está listada entre las competencias profesionales del médico.

En la escala de institución, el profesionalismo se puede reconocer en diversos enunciados institucionales y las competencias profesionales transversales solo en el perfil del egresado. En relación con los programas de estudio, las dos competencias menos seleccionadas corresponden al componente Comunicación, en particular, trata de la relación con el paciente y/o la familia.

En relación con las encuestas, además de los atributos (ver nota: b) relevados sobre profesionalismo (cuyos resultados se presentan en la discusión) se indagó su enseñanza. Para el 45.7 $\%$ de los estudiantes se enseñan todos, un $49.6 \%$

Revista Methodo: Investigación Aplicada a las Ciencias Biológicas. Universidad Católica de Córdoba. Jacinto Ríos 571 Bo Gral. Paz. X5004FXS. Córdoba. Argentina. Tel.: (54) 3514517299 / Correo: methodo@ucc.edu.ar / Web: methodo.ucc.edu.ar | ARTICULO ORIGINAL Rev. Methodo 2020;5(1):09- 
solo algunos y un $3.9 \%$ afirma que no se enseñan. En tanto para profesionales el porcentaje es sensiblemente menor, para el $28 \%$ enseñan todos, un $53 \%$ algunos y el $15 \%$ dice no se enseñan. Tanto los profesionales como los estudiantes, señalan que se aprenden con el ejemplo, en el contacto con pacientes con demostraciones $\mathrm{y}$ mostraciones.

En lo relativo a cómo se evalúa, para los estudiantes en primer lugar mediante prácticas con pacientes $(31.3 \%)$ y mediante observaciones de las prácticas $(21.2 \%)$ que incluyen simulaciones $(4 \%)$. En tercer lugar, con instrumentos de evaluación centrados -en mayor medida- en los conocimientos como pruebas de opciones múltiples (POM), $17.2 \%$, pruebas de desarrollo y exámenes orales (16.2\% cada uno). También se incluye la evaluación continua $(9.1 \%)$. Para una quinta parte no se evalúa $(21 \%)$ o por no sabe $(9.1 \%)$. Para los profesionales, en primer lugar, están las prácticas con pacientes $(29.7 \%)$. La mayor diferencia se encuentra en simulaciones a la que le asignan un $24 \%$. Estas respuestas se distancian de las dadas por los estudiantes (para quienes solo $4 \%$ alude a simulaciones). Los instrumentos de evaluación centrados en los conocimientos fueron elegidos menor medida: pruebas de desarrollo $(6.6 \%)$, POM $(5.5 \%)$ y exámenes orales $(3.3 \%)$. En cambio, se incluyeron otros como casos y problemas (16.5\%), actividades teórico-prácticas (12.1\%), situaciones $(11 \%)$, talleres de procedimientos $(6.6 \%)$, escalas (3.3\%), miniCEX (3.3\%), Examen Clínico Objetivo Estructurado (ECOE), proyectos comunitarios, proyectos sociales, ejercicios con $2.2 \%$ cada uno, Portafolio y rúbricas. En relación con la evaluación continua y no sabe recibe obtiene un $6.6 \%$ y no se evalúa la quinta parte $(4.4 \%)$. Nuevamente, los docentes muestran un mayor conocimiento de otros modos en que es posible evaluar profesionalismo, pero al no ser conocido por los estudiantes se abre el interrogante si solo esto se reduce solo conocimiento o bien se concreta en las prácticas de enseñanza y evaluación.

Ahora bien, en relación con las competencias profesionales transversales, para los estudiantes se enseñan mediante prácticas clínicas (38.6\%) y exposiciones $(31.7 \%)$. Esto último permite suponer que los estudiantes ubican esta enseñanza en forma teórica. También mediante prácticas de integración (31.7\%) y con el ejemplo (23.8\%). Nuevamente se incluye: "no es enseñable" en estudiantes $(4.1 \%)$ y profesionales $(6.1 \%)$.

En relación con la evaluación de estas competencias, según los estudiantes se concreta mediante observación de las prácticas $(23.5 \%)$ y con pacientes $(19.4 \%)$, mediante pruebas (de desarrollo $12.2 \%$, POM $12.2 \%$, y orales $11.2 \%$ ).
También en este caso se alude a una evaluación centrada -en mayor medida- en la teoría. En la enseñanza el ejemplo tiene un peso mayor (23.8\%) que el asignado en la evaluación (9.2\%), lo mismo sucede con las actividades de integración (18\% y en enseñanza $9.2 \%$ ) y las simulaciones $(11.2 \%$ y en enseñanza $6.1 \%$ ).

Según profesionales, en primer lugar, se encuentra, al igual que en estudiantes, observación de las prácticas (39.4\%) y con pacientes (27.3\%). También la simulación, aunque con mayor peso $(19.2 \%)$ y las pruebas (de desarrollo $13.1 \%$, orales $11.1 \%$, POM $8.1 \%$ ). Ofrecen una diversidad de alternativas: casos y problemas $(14.1 \%)$, reflexión sobre la práctica $(8.1 \%)$, ejercicios $(7.1 \%)$, escalas (6.1\%), ECOE/debrifin (4\%), encuestas (3\%), evaluación integral de la práctica $(3 \%)$, portafolio (3\%), autoevaluación (2\%), entrevistas (2\%), miniCEX (1\%), workshops (1\%). No figuran las actividades de integración como una modalidad de evaluación. Finalmente, también aparece "no se evalúa" tanto en estudiantes $(10.2 \%)$ como en profesionales $(6.1 \%)$.

De este modo, las respuestas de estudiantes y profesionales difieren en particular en relación con la diversidad de propuestas que señalan los segundos en mayor medida que los primeros. Además, al remitir a propuestas sostenidas en la teoría en mayor medida cabe el interrigante acerca de en qué medida las competencias, que se espera se desarrollen como prácticas en situación, son abordadas de este modo.

\section{Discusión}

Los estudiantes generaron 51 categorías para atributos (ver nota: c) del profesionalismo. Los seleccionados en mayor medida fueron: responsabilidad (ver nota: d) $(40.3 \%)$, respeto $(37.2 \%)$ y humanismo médico $(35.7 \%)$, cerca del $30 \%$ : empatía $(29.5 \%)$ y buen trato con los otros $(28.7 \%)$. En el caso del humanismo, se trata de un atributo que se encuentra entre aquellas características esperadas por la institución. En quinto lugar: conocimientos $(21.7 \%)$ y en sexto una competencia que es mencionada con frecuencia en toda la literatura y que además figura en la Resolución $\mathrm{N}^{\mathrm{o}} 1314^{13}$-aunque no como capacidad-, y capacidad de trabajo en equipo (16.3\%). Luego, confidencialidad, humildad y compromiso (13.2 \%), y cercano al $10 \%$, actualización y ética (11.6\%) Si se lo contrasta con la investigación realizada por otros autores con estudiantes $^{16}$, estos generaron 58 atributos y comparten con las respuestas de estudiantes de la Universidad pequeña de reciente creación (UF), la obtención de valores más altos en: empatía,

Revista Methodo: Investigación Aplicada a las Ciencias Biológicas. Universidad Católica de Córdoba. Jacinto Ríos 571 Bo Gral. Paz. X5004FXS. Córdoba. Argentina. Tel.: (54) 3514517299 / Correo: methodo@ucc.edu.ar / Web: methodo.ucc.edu.ar | ARTICULO ORIGINAL Rev. Methodo 2020;5(1):0914. 
responsabilidad, respeto y capacitación continua (que en este caso figura como actualización).

Los profesionales generaron 74 categorías sobre profesionalismo. La mitad de las respuestas se ubican en actualización como su principal atributo (ver nota: e). Esto está en consonancia con la investigación desarrollada por otros autores ${ }^{16}$, quienes lo relavaron en profesionales médicos y obtuvieron como segundo atributo: capacitación continua. Además, coincide en que lo asignado como de mayor relevancia: honestidad, empatía, amabilidad, ética, compromiso, responsabilidad, aunque no así excelencia. Y con la investigación de Gutiérrez Gutiérrez ${ }^{17}$ sobre los atributos de los médicos internos de respeto, altruismo (aunque en este caso su valoración fue menor), responsabilidad, no así con justicia y excelencia que fueron elegidos en esa investigación.

La afirmación de que el profesionalismo se trae de la casa o de la vida, es decir, se asocia este constructo con aquello que se aprende en forma ubicua que se desarrolla en la dimensión personal difieren de las presentadas por otros ${ }^{18}$, quienes le otorgan un peso sustantivo al proceso educativo.

\section{Conclusión}

Las competencias profesionales transversales y el profesionalismo oficiaron de puerta de entrada para abordar la profesionalización de los futuros médicos y médicas. Para lograrlo, resulta necesario introducir modificaciones en todas las escalas. Para ello, se propone, llevar adelante un trabajo colaborativo y colegiado la redefinición de estándares a fin de incluir, en forma temprana, a los estudiantes en espacios compartidos, con estudiantes de las carreras de salud, tanto en servicios de salud como en comunitarios. A partir de esos cambios, abordar las modificaciones en las otras escalas. Este trabajo no puede reducirse a en las instituciones formadoras, ni concretarse en dos tiempos (primero la educación y luego los servicios de salud) sino en forma conjunta, desde un enfoque sociocomunitario. De este modo, se procura que el futuro profesional se inserte en situaciones reales, en situaciones de trabajo cooperativo con otros, en lugar de que la centralidad recaiga sobre el desempeño individual y su accionar se reduzca a solucionar problemas. Se trata de un trabajo demandará tiempo y un fuerte compromiso ético.

\section{Notas:}

a. La producción y reproducción de significados está tomado de Grazier (2010, p.75). El autor emplea el término "significados", que aluden a la construcción sociodiscursiva en lugar de "sentidos" que hacen referencia a la construcción individual. En adelante se usará desde este enfoque.

b. La RAE define atributo como: "las propiedades, características o cualidades de un ser". Se ha optado por este término en tanto se consideró que era posible que fuera comprendido en forma similar tanto por estudiantes como por profesionales.

c. Esta variable tiene un tratamiento diferente porque podían elegir más de una opción. Por ello el total de casos no hace referencia a los encuestados sino a las respuestas dadas.

d. Se utiliza itálica en lugar de comillas para facilitar la lectura

e. Esta variable tiene un tratamiento diferente porque podían elegir más de una opción. Por ello el total de casos no hace referencia a los encuestados sino a las respuestas dadas.

\section{Bibliografía}

1. Lotito, F. (21 de septiembre de 2017) Mala Praxis Médica. Negligencias y errores médicos: un grave problema de salud bajo observación.WorldDiagnisticsWord.

Recuperado de: https://www.diagnosticsnews.com/rpnews $2 / 2$ 5962-mala-praxis-medicanegligencias-yerrores-medicos-un-grave-problema-desalud-bajo-observacion.

2. Ramos Domínguez, B. N. (2005). Calidad de la atención de salud: Error médico y seguridad del paciente. Revista Cubana de Salud Pública. 31 (3), pp. 239-244. Recuperado de: http://scielo.sld.cu/pdf/rcsp/v31n3/spu10305. pdf.

3. Rodríguez de Castro, F (2012) Proceso de Bolonia (V): el currículo oculto. Educación Médica; 15 (1), pp. 13-22. Recuperado de: https://www.um.es/c/document_library/get_fi le?uuid=b0108286-4545-4d7b-a294c4c8df41c5ef\&groupId=115466.

4. Ruiz de Gauna, P, González Moro, V. y Morán-Barrios, J. (enero-marzo de 2015). Diez claves pedagógicas para promover buenas prácticas en la formación médica basada en competencias en el grado y en la especialización. Educación Médica. 16 (1), p. 34-42. Recuperado de: http://ac.elscdn.com/S1575181315000078/1-s2.0- 
S1575181315000078-

main.pdf? tid=a6380dd0-cdc5-11e5-961100000aacb361\&acdnat $=1454868628 \_2 \mathrm{~d} 3 \mathrm{e} 02$ 4fb3d3a2d420178eea85777140

5. Vera Carrasco, O. (2015). El rediseño curricular por competencias: un reto para nuestra Facultad de Medicina. Revista Cuadernos, 56 (2), pp. 7-8. Recuperado de: http://www.revistasbolivianas.org.bo/pdf/chc /v56n2/v56n2_a01.pdf.

6. Baños, J.E. y Pérez J. (2005) Cómo fomentar las competencias transversales en los estudios de Ciencias de la Salud: una propuesta de actividades. Educación Médica, 8 (4), pp. 216-225. Recuperado de: http://www.upf.edu/biomed/_gavi/comptrans. pdf.

7. Etcheverry, G. J. (2000). La facultad de Medicina en la formación de pregrado. Ponencia. II Jornadas de APS. CONAMER. Universidad Nacional de La Plata. La Plata, Argentina, pp. 131-196. Recuperado de: http://www.unla.edu.ar/documentos/institutos /isco/cedops/libro1a12.pdf.

8. Terigi, F. (1999). Curriculum. Itinerarios para aprehender un territorio. Buenos Aires, Argentina: Santillana.

9. Corominas Rovira, E. (2001). Competencias genéricas en la formación universitaria. Revista de educación, (325), p. 299-321. Recuperado http://www.mecd.gob.es/dctm/revista-deeducacion/articulosre325/re3252109962.pdf? documentId=0901e $72 \mathrm{~b} 8125 \mathrm{de} 0 \mathrm{e}$.

10. González Maura, C y González Tirados, R. M. (mayo-agosto de 2008). Competencias genéricas y formación profesional: un análisis desde la docencia universitaria. Infancia y escuela. (47), pp. 183-209. Recuperado de: http://www.rieoei.org/rie47a09.pdf.

11. Bourdieu, P. (mayo de1981) Describir y prescribir. Notas sobre las condiciones de posibilidad y los límites de la eficacia política Recherche en Sciences Sociales, 38, pp. 6973. Traducción de David Velasco Yáñez. Recuperado de: https://davidvelasco.files.wordpress.com/200 9/01/describir-y-prescribir.pdf.

12. Ministerio de Cultura y Educación (10 de agosto de 1999). Resolución Ministerial $N^{\circ}$ 535. Buenos Aires, Argentina.

13. Ministerio de Educación, Ciencia y Tecnología. (4 de septiembre de 2007).
Resolución Ministerial $\mathrm{N}^{\mathrm{o}}$ 1314. Buenos Aires, Argentina.

14. Stake, R. (1999). Estudio de Caso. Madrid, España: Morata.

15. Yin, R.K. (1994). Case Study Research. Design and Methods. Applied Social Research Methods, 5. London, England: Sage Publications. Recuperado de: http://www.madeiraedu.pt/LinkClick.aspx ?fileticket=Fgm4GJW VTRs\%3D\&tabid=3004.

16. Fasce, E., Echeverría, M., Matus, O., Ortiz, L., Palacios, S., y Soto, A. (2009). Atributos del profesionalismo estimados por estudiantes de medicina y médicos. Análisis mediante el modelo de disponibilidad léxica. Revista Médica de Chile, 137(6), p. 746-752. Recuperado de https://scielo.conicyt.cl/scielo.php?script=sci _arttext\&pid=S0034-98872009000600003.

17. Gutiérrez Gutiérrez, C. (2013). Percepción de internos y residentes de aspectos del profesionalismo médico en establecimientos de salud Lambayeque 2013. Tesis de Maestría. Escuela de Postgrado de la Universidad Católica Santo Toribio de Mogrovejo. Chiclayo, Perú. Recuperado de http://tesis.usat.edu.pe/jspui/bitstream/12345 6789/184/1/TM_Gutierrez_Gutierrez_Carme nIsabel.pdf.

18. Sogi, C., Cárdenas, M, Zavala, S, Mendoza, A. y Jiménez, M.R. (2015). El profesionalismo en la formación del médico en la Universidad Nacional Mayor de San Marcos. Un estudio de sílabos. Anales de la Facultad de Medicina, 76 (4), pp. 339- 348. Recuperado de http://www.redalyc.org/pdf/379/3794342900 4.pdf

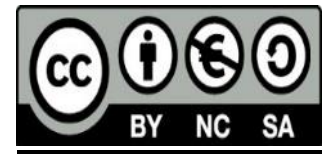

